\title{
Flexible Piezoelectric Drop-On-Demand Droplet Generation
}

\author{
Norbert Riefler*1, Thomas Wriedt ${ }^{2}$, Udo Fritsching ${ }^{1}$ \\ ${ }^{1}$ Institute of Material Science (IWT), Bremen, Germany \\ ${ }^{2}$ Particle and Process Engineering, University Bremen, Germany \\ *Corresponding author: riefler@iwt.uni-bremen.de
}

\begin{abstract}
The size of droplets generated by piezoelectric drop-on-demand (DOD) droplet generators can be varied to a certain degree within one order of magnitude. This variation means that the droplet size is not solely determined by the nozzle diameter, and the droplet generation process is not restricted to drops extruded through a nozzle in conventional operation. By varying the electronic driving pulse, different droplet sizes can be obtained. To investigate the interaction of piezoelectric pulse excitation and the finally produced droplets, different approaches are applied. A comparison of a modal analysis of a pure piezo based on mechanical admittance calculations proofs the usability of electrical impedance measurements. This kind of measurements are then compared to finite-element simulations of a coupled piezo system - one as actuator, the other as pressure sensor - to extend the usable methods with the result that the fluid is of minor influence on the modal frequencies. Last, two phase fluid flow simulations with consequent pressure wave evaluations of the fluid show different pressure wave frequency specta than the modal analysis.
\end{abstract}

\section{Keywords}

Piezoelectic droplet-on-demand droplet generator, modal analysis, compressible two-phase flow

\section{Introduction}

Piezoelectric droplet generators are used commercially in inkjet printers as well as scientific instruments to, e.g., investigate single droplet phenomena like combustion [1] or drop collisions [2]. For controlled production of monodisperse droplets, drop-on-demand offer the possibility of generating droplets of different size solely by applying appropriate electrical signals to the piezoelectric actuator in 'w'-waveform [3] or in the form of staircase voltages [4]. Temple [5] suppossed that droplets can be generated with a much smaller diameter than the nozzle orifice and this is related to a 'Faraday Resonance', i.e. a resonance phenomenon that occurs in vertical oscillation of a column of liquid with a free surface. These surface wave resonances are a consequence of a pressure rise near the nozzle and lead to a transfer of energy and momentum from the acoustic field to the droplet.

The generation of droplets smaller than the nozzle orifice is of interest, e.g., to produce small droplets of a suspension. In this case, typical DOD droplet generators with a nozzle diamater well below $70 \mu \mathrm{m}$ tend to clogg with the consequence of a laborious cleaning process. Therefore, a dismountable droplet generator is developed to enable simple cleaning. Additionally, the nozzle diameter is increased to $200-300 \mu \mathrm{m}$ which is unusual for common fluids like water [6]. The generation parameters (i.e. pulse voltage and pulse time) for fluids with different viscosities and surface tension is experimentally tested. The transfer of momentum of a piezo actuator to the tube structure in the way of a modal analysis is also investigated and delivers preferred excitation frequencies of the solid tube part to the fluid. Last, the response of a compressible fluid to a pressure pulse is analyzed and interpreted as pressure waves by Fourier transformation.

\section{DOD generation experiments}

A principal sketch of a piezoelectric droplet generator can be seen in figure 1. It consists of a titanium tube with screw threads on each end, see figure 2, top, shown in upward directed operation in the bottom of this figure. In an experimental series, fluids with varying viscosities $\eta$, densities $\rho$ and surface tensions $\sigma$ given in table 1 are used.

Table 1. Fluid properties and generated droplet diameters

\begin{tabular}{lcccc}
\hline & $\eta[\mathrm{mPa} \cdot \mathrm{s}]$ & $\rho\left[\mathrm{kg} / \mathrm{m}^{3}\right]$ & $\sigma[\mathrm{N} / \mathrm{m}]$ & $d_{\text {drop }}[\mu \mathrm{m}]$ \\
\hline DI water & 1 & 1000 & 0.0725 & $24 \ldots 170$ \\
\hline water-glycerol & 2 & 1054.35 & 0.0715 & $135 \ldots 180$ \\
\hline ethanol-glycerol & 3 & 867.67 & 0.0225 & $113 \ldots 130$ \\
\hline
\end{tabular}




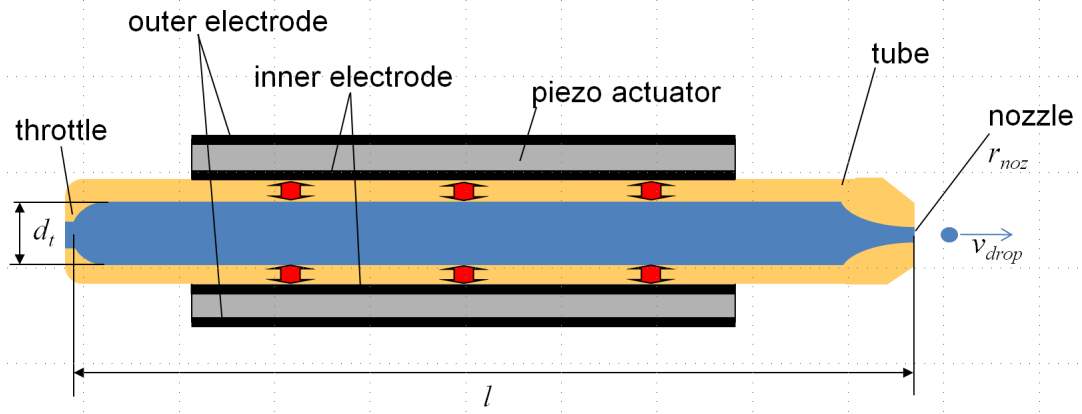

Figure 1. Sketch of a piezelectric droplet generator; the actuator moves the tube in radial direction (displacement $\leq 100 \mathrm{~nm}$ ).

The generated monodisperse and - at least one hour - stable droplets show different diameters and velocites. The dimensionless Reynolds and Ohnesorge numbers:

$$
R e=\frac{\rho d v_{d r o p}}{\eta}, \quad O h=\frac{\sqrt{W e}}{R e}=\frac{\eta}{\sqrt{\sigma \rho d}}
$$

are calculated based on these data to characterize the operating regime [7] of the droplet generator. The findings are shown in figure 3 together with the principal limits. The droplet diameters vary with driving signal in particular with DI water almost one order of magnitude and emphasize the flexibility of piezoelectric droplet generators.

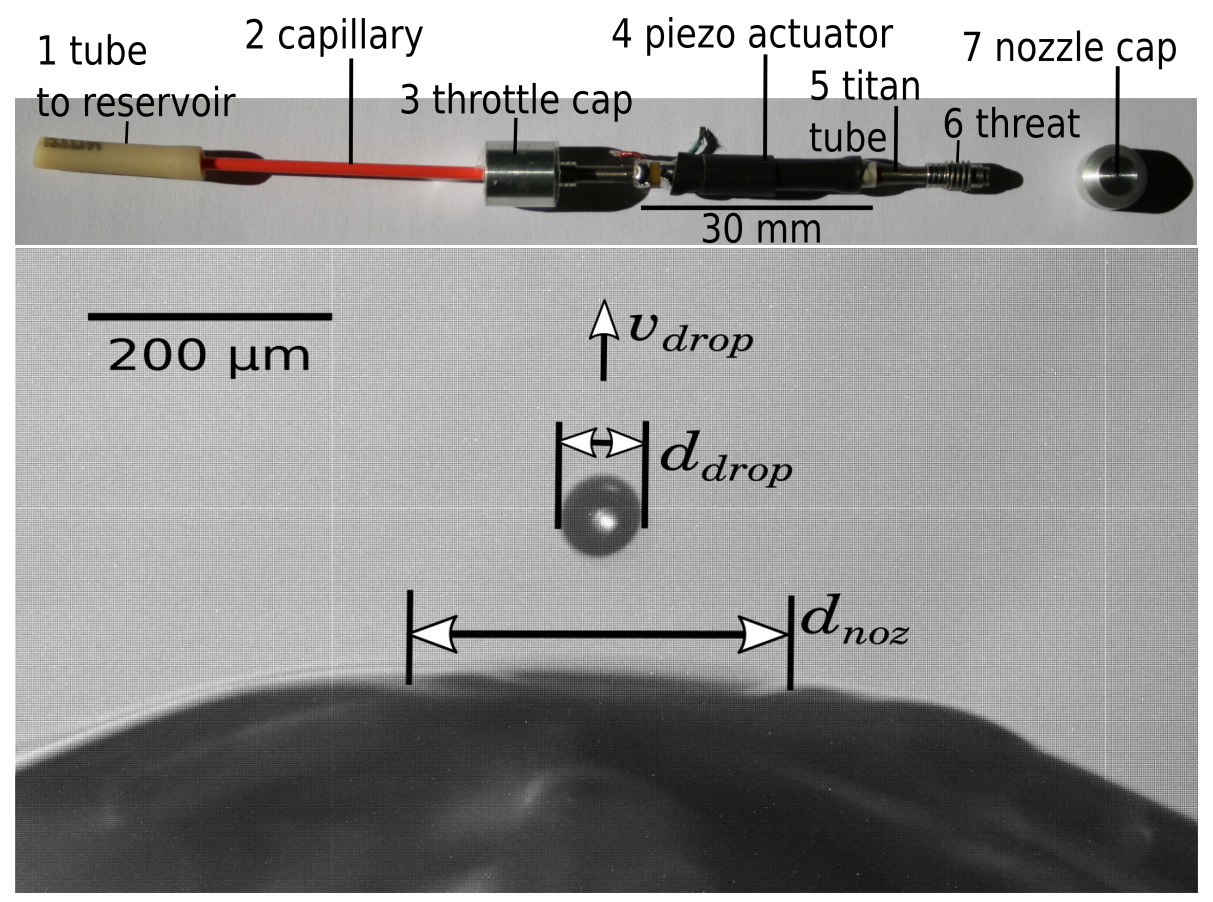

Figure 2. Droplet generator with the screwed off nozzle (7, top right) and piezo actuator in the middle (4); the red PEEK capillary

(2) on the left can be moved into the titan tube (5); image of a droplet with diameter of $d_{d r o p}=70 \mu \mathrm{m}$ from a nozzle with diamater of $d_{n o z}=300 \mu \mathrm{m}$ (below).

The restriction to a few stable operating points of the droplet generator for the ethanol-glycerol mixture is due to the low contact angle of this fluid with many solid materials. This results in a strong tendency to nozzle wetting which leads to a unstable generation process. The reason of the low number of operating points in case of deionized water is mainly due to a restricted maximum voltage of the used power supply of $\Delta V=200 \mathrm{~V}$. 


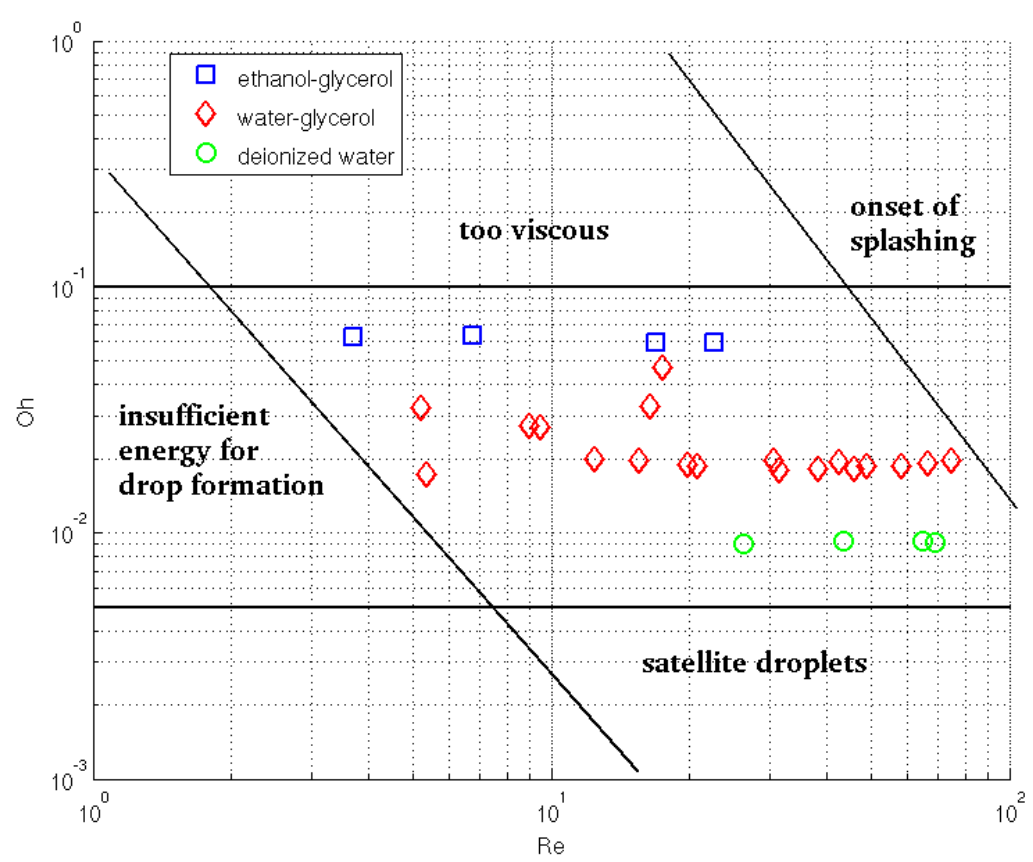

Figure 3. Stable droplet regime of the used DOD device.

\section{Modal analysis \\ One piezo system}

The piezoelectric actuator is characterized using a vector network analyzer (VNA) in reflection mode to investigate the electrical response of the system to different frequencies of the electrical drive signal. A low voltage sine signal with frequency range from about $10 \mathrm{kHz}$ to $1 \mathrm{MHz}$ is applied first to the piezo alone and in a second measurement to the piezo bonded to the titan tube. These measurements correspond to the excitation with a Dirac delta pulse and are equivalent to the impulse response function. At resonance frequencies strong responses can be observed, and these eigenfrequencies are the modes of the acousto-mechanical system [8]. From analytical considerations of a finite cylindrical beam with the properties given in table 2 the mechanical admittance can be expressed by modal summation [8]:

$$
Y(\omega)=i \omega \sum_{n=1}^{\infty} \frac{\phi_{n}(x)^{2}}{M\left(\omega_{n}^{2}\left(1+i \eta_{\text {loss }}\right)-\omega^{2}\right.}
$$

with the angular frequency $\omega=2 \pi f$, the modal mass $M=m l$ with the mass per unit area $m=\rho 2 \pi a h$, the mean radius of the shell $a=\left(d_{o}+d_{i}\right) / 2$, the shell wall thickness $h=\left(d_{o}-d_{i}\right) / 2$, the second moment of area (areal moment of intertia) $I=\pi a^{3} h$, the damping coefficient $\eta_{l o s s}$, and the modal function of $n$-th order:

$$
\phi_{n}(x)=\sqrt{2} \sin \left(\frac{n \pi x}{l}\right)
$$

for any position $x$ along the beam and $n=1,2, \ldots$. The natural frequencies are given by:

$$
\omega_{n}=\frac{n^{2} \pi^{2}}{l^{2}} \sqrt{\frac{E I}{m}}
$$

and they agree with the modal frequencies of a cylindrical beam. However, due to mechanical constraints, not all modal frequencies can be excited. In our case, a voltage applied to the piezoelectric actuator excites the coupled tube-piezo system mainly in radial direction, so it is expected that not every mode is experimentally observable.

The measured electrical impedances $Z$ are compared to the mechanical admittances $Y$ calculated by eqn. (2) because the admittance of a lumped mass-spring system corresponds to the impedance of a corresponding electrical equivalent, see Firestone [9]. However, not every acousto-mechanical mode contributes to the admittance spectrum. Only by using modes number $n=1,2,6$ and 21 the admittance spectrum shown in figure 4 with an estimated damping factor of $\eta=0.02$; lower damping inflates the calculated peaks while larger values overdamp the admittance curve. 
Table 2. Geometrical and material properties of the used lead circonate titanate piezoelectric material PIC151 of length $l$, inner diameter $d_{i}$, outer diameter $d_{o}$, Youngs modulus $E$ and density $\rho$

\begin{tabular}{ccccc}
\hline$l[\mathrm{~mm}]$ & $d_{i}[\mathrm{~mm}]$ & $d_{o}[\mathrm{~mm}]$ & $E[\mathrm{GPa}]$ & $\rho\left[\mathrm{kg} / \mathrm{m}^{3}\right]$ \\
\hline 30 & 2.2 & 3.2 & 10 & 7800 \\
\hline
\end{tabular}

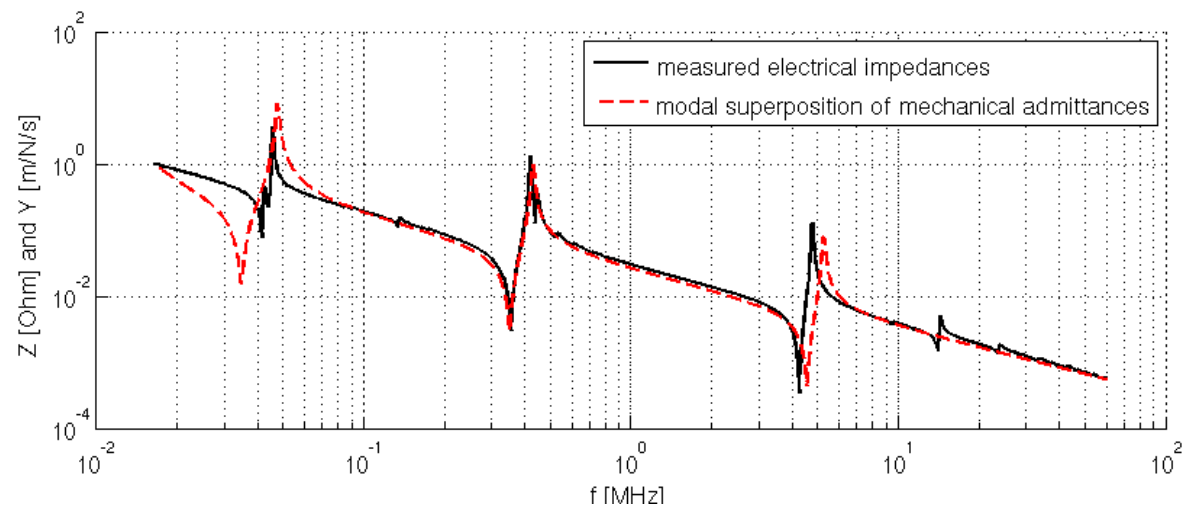

Figure 4. Comparison of measured impedance $Z$ with calculated admittance spectra $Y$.

This modal analysis considers the piezo tube only. As far as the piezo is adhesively bonded to the titan tube, the electrical impedance spectra changes as shown in figure 5.

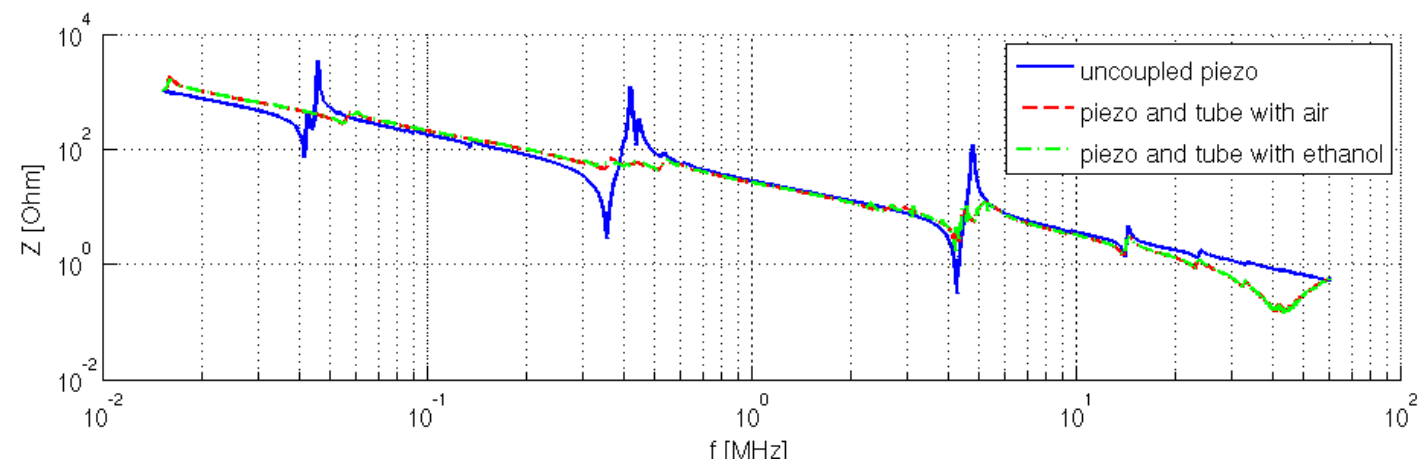

Figure 5. Measured impedance spectra $Z$ for the umcoupled piezo (blue, straight line) and the coupled system with air (red, dashed line) and filled with ethanol (green, dash-dotted line).

The tube strongly damps the pronounced modes of the uncoupled piezo (see the dashed and dash-dotted lines). However, the resonance frequencies changes only in case of the first resonance from about $42 \mathrm{kHz}$ to $55 \mathrm{kHz}$, and a new resonance at $\approx 15 \mathrm{kHz}$ (leftmost) occurs. The kind of fluid within the tube seems to be of minor importance to the system as can be seen from the curves with air and ethanol as fluid, that are only slightly different.

\section{Two coupled piezos}

The effect of the fluid - either air or ethanol - of the modal analysis on the droplet generator is small because the response is dominated by the solid materials titan and the PZT piezo ceramic. However, the influence of the mechanical vibrations on the fluid is crucial for a descrption of the fluidal droplet generation process. Therefore, it is intended to perform a fully coupled fluid-structure interaction simulation based on the finite element method (FEM). This will replace the analytical approach, and the reliability of the FEM simulations is therefore proved using a specical double piezo system, where the mechanical response function can be compared to an electrical stimulated excitation by a piezo actuator on the titan tube. I.e., the recording of the response on a second piezo, also bonded to the same titan tube, serves as a mechanical sensor.

The mesh of the FEM system, generated by the 'DesignModel' of ANSYS ${ }^{\odot}$ Academic Research, Release 17.2 within the Workbench framework, is shown in figure 6. A modal analysis is performed and compared to the resonance spectrum of the electrical driven system, where one piezo is fed with a sine wave of $U=5 \mathrm{~V}$ and the second piezo is connected to an oscilloscope for visualization and measurement of voltage. The resonance frequencies are determined at the local maximum of the piezo sensor signal in the frequency domain. 


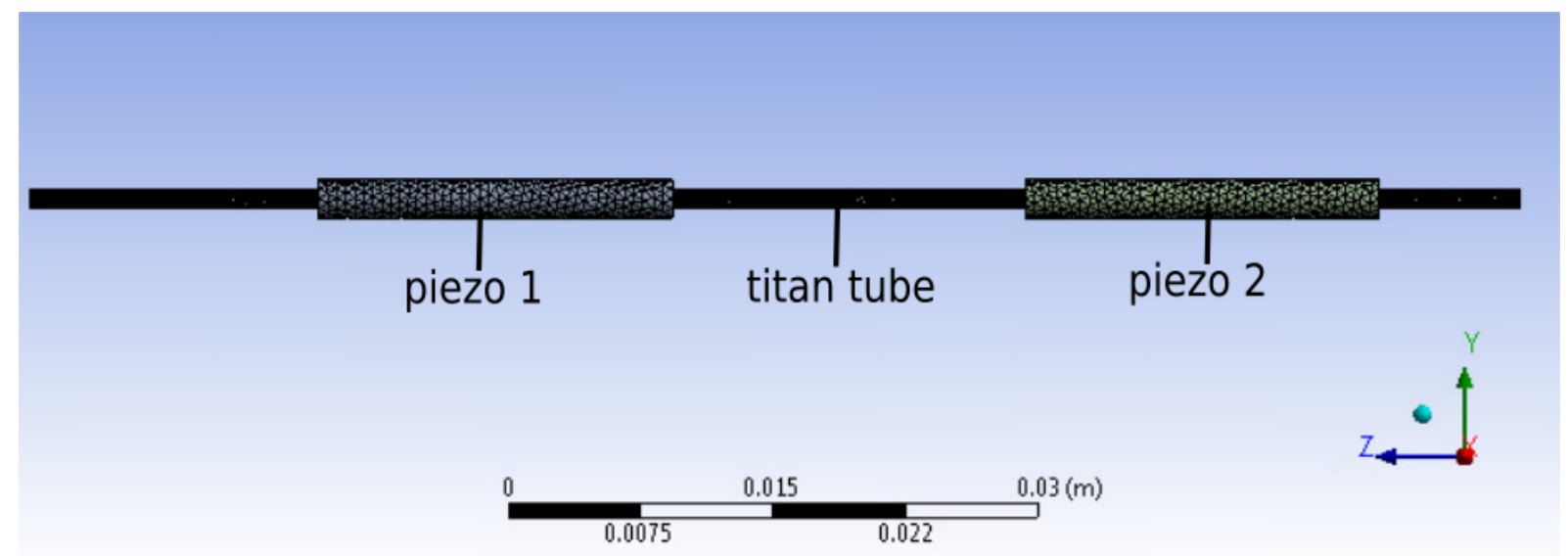

Figure 6. FEM model of a coupled system with two piezo actuators.

The modal frequencies from the FEM modal analysis and the frequencies at the resonance peaks show a strong similarity, although some values in the sweep test are missing. This can be explained by the large outputs of the piezo sensor in the frequency range starting from about $50 \mathrm{kHz}$ up to about $110 \mathrm{kHz}$. However, the results indicate that the FEM modal analysis is sufficiently precise and can be used for system characterizations.

Table 3. Comparison of modal frequencies from FEM analysis $\left(f_{F E M}\right)$ and measured frequencies of peak signals $\left(f_{\text {sweep }}\right)$

\begin{tabular}{ccccccccccc}
\multicolumn{6}{c}{ during frequency sweep; empty fields mean that there is mean no maximum detectable. } \\
\hline$f_{F E M}$ & 10.5 & 12.1 & 14.4 & 16.2 & 23.1 & 25.6 & 33.0 & 33.1 & 41.9 & $\mathrm{kHz}$ \\
$f_{\text {sweep }}$ & 8.1 & 12.7 & 13.2 & 17.5 & 23.5 & 28.5 & 32.3 & 33.5 & & $\mathrm{kHz}$ \\
\hline$f_{F E M}$ & 45.2 & 53.9 & 59.7 & 65.9 & 66.0 & 66.6 & 68.2 & 72.7 & 75.2 & $\mathrm{kHz}$ \\
$f_{\text {sweep }}$ & 44.3 & - & - & 63.0 & 63.2 & 66.6 & 67.2 & - & - & $\mathrm{kHz}$ \\
\hline$f_{F E M}$ & 78.4 & 85.6 & 88.1 & 92.7 & 92.8 & 101.5 & 113.2 & 117.3 & & $\mathrm{kHz}$ \\
$f_{\text {sweep }}$ & - & - & - & - & - & - & 115.3 & - & & $\mathrm{kHz}$ \\
\hline
\end{tabular}

\section{Compressible two phase flow}

The fluid cell of the droplet generator employed above is mapped as a 2D simulation case with cylinder symmetry in OpenFOAM, see figure 7. The transient simulation is performed with a compressible solver applying a volume of fluid method for the two phases water and air ('compressiblelnterFoam'). A pressure inlet in place of the piezo actuator (indicated by the black line in figure 7) is charged with a trapezoidal pressure pulse. The symmetry line is on the bottom and except the right inlet-outlet atmosphere patch, all other boundaries are walls.

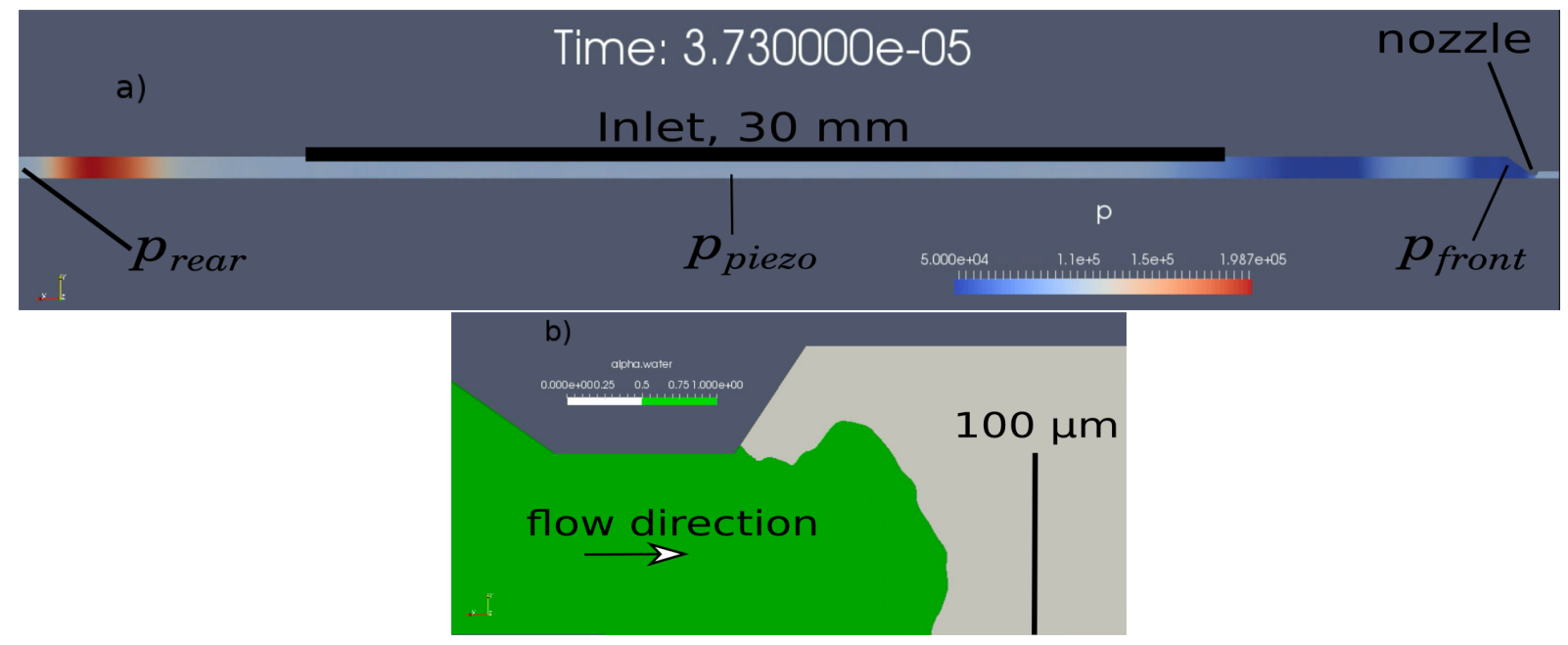

Figure 7. Two phase flow simulation with a compressible solver; a) pressure distribution along the fluid cell at $t=37.3 \mu$ s after start of the pressure pulse of length $t_{\text {pulse }}=20 \mu \mathrm{s}$ at the inlet (indicated with the black line); b) extruded fluid (green) with waves on the surface; orifice, i.e. nozzle radius $r_{n o z}=100 \mu \mathrm{m}$.

A pressure pulse of length $t_{p u l s e}=20 \mu \mathrm{s}$ and pressure increase of $\Delta p=1$ bar (additionally to the $1013 \mathrm{mbar}$ ambient pressure) excites the fluid in the chamber of figure 7 to oscillate at several frequencies. There is no 
response on the water-air interface on the nozzle until about $t=30 \mu \mathrm{s}$, but then water starts to extrude and a large droplet is generated. The free surface shows slight oscillations, however no Faraday resonances - proposed by Temple [5] - are observable due to the 2D mesh geometry of the simulations. Furthermore, the extruded fluid volume yields a droplet greater than those from experimental findings given in table 1.

To investigate the pressure values within the fluid cell in time, three pressure recording probes - positioned in the middle close to the piezo $\left(p_{\text {piezo }}\right)$, in the front cone close to the nozzle $\left(p_{\text {front }}\right)$ and in the rear $\left(p_{\text {rear }}\right)-$ are represented in figure 8 . The applied pressure pulse at the inlet can be clearly seen together with some overshoots (blue, straight line). At about $12 \mu$ s the pressure wave arrives simultaneously at the front cone and the rear. However, due to the conical constriction close to the nozzle on the right, the pressure wave form differ from the rectangluar rear side (left) with the result of different pressure curves.
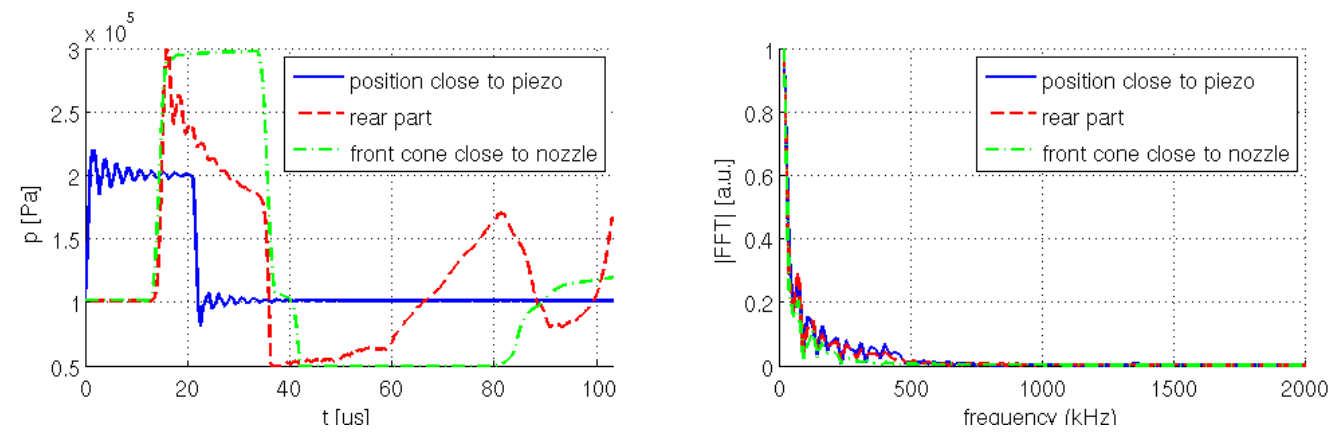

Figure 8. Pressure values at three probes located along the fluid cell after a pulse excitation length of $t_{\text {pulse }}=20 \mu \mathrm{s}$ (left); FFT's of the pressure waves (right).

If the pressure pulse is shorten to a triangular pulse, where the pressure rises up to $\Delta p=1$ bar at $\Delta t=1 \mu \mathrm{s}$, and falls afterwards back to ambient pressure at $\Delta t=1 \mu \mathrm{s}$ again, the response at the same probe locations delivers different curves, see figure 9.
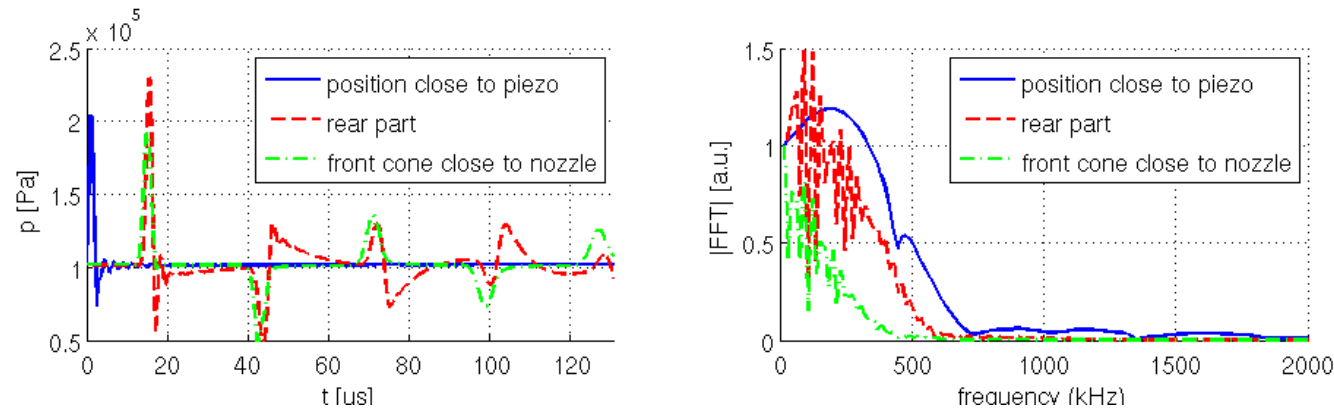

Figure 9. Same as in figure 8 but $t_{p u l s e}=2 \mu \mathrm{s}$.

Each pressure curve given in the left parts of figures 8 and 9 are Fourier transformed and represented in the right part. Therefore, the short pulse $\left(t_{\text {pulse }}=2 \mu \mathrm{s}\right)$ triggers a much larger frequency spectrum of pressure waves than the longer pulse. A thorough evaluation of the pressure response in time at about 1000 different probe positions distributed within the fluid cell serves as data basis for an evaluation of the frequency response of the fluid cell, where the Fourier transformed pressure function of each probe is stored. Some of these resonance frequencies appear many times and their occurence are counted.

To clarify if the the pressure wave frequencies and their number of counts can be reflected by the resonances in the mechanical impedance spectra of figure 5 , both results are represented in figure 10 . Every frequency is projected to the measured impedance value, and the number of counts, i.e. how often this frequency occurs in the FFTs of the pressure waves, are represented by the size of the dots. It can be seen that the longer excitation pulse $\left(t_{\text {pulse }}=\right.$ $20 \mu \mathrm{s}$, see figure 8) excites a lower frequency spectra, however with more pronounced single resonances, while the shorter pulse $\left(t_{\text {pulse }}=1 \mu \mathrm{s}\right.$, see figure 9$)$ excites a much broader spectra. However, the mechanical vibrations, i.e. the eigenmodes of the mechanical (solid) coupled piezo-tube system, are not represented by the fluid flow simulations. This means that the pressure waves within the fluid cell are not related to the mechanical piezo-tube vibrational system, a result found already by the impedance spectra, see figure 5 .

\section{Conclusions}

Analytic and FEM simulations are done to infer the eigenmodes of the solid material of the investigated droplet generators showing good agreement of the peak resonances of the piezo. Further comparisons of FEM simulations with electrical measured resonances on a system with two piezos coupled via a titan tube show also good agree- 
ment. These results permit the usage of the different methods to investigate the behaviour of the droplet generator which is essentially dominated by the coupling of the piezo and the tube and to a minor degree the coupling with the liquid in the tube chamber.

Additionally performed compressible and multiphase fluid flow simulations show rich spectra of pressure wave frequencies, however they cannot be directly attributed to the mechanical modes. Therefore, a complete fluidstructure-interaction model is actually developed to see the influence of the mechanical vibrations on the fluid and the generation of droplets in DOD generator.
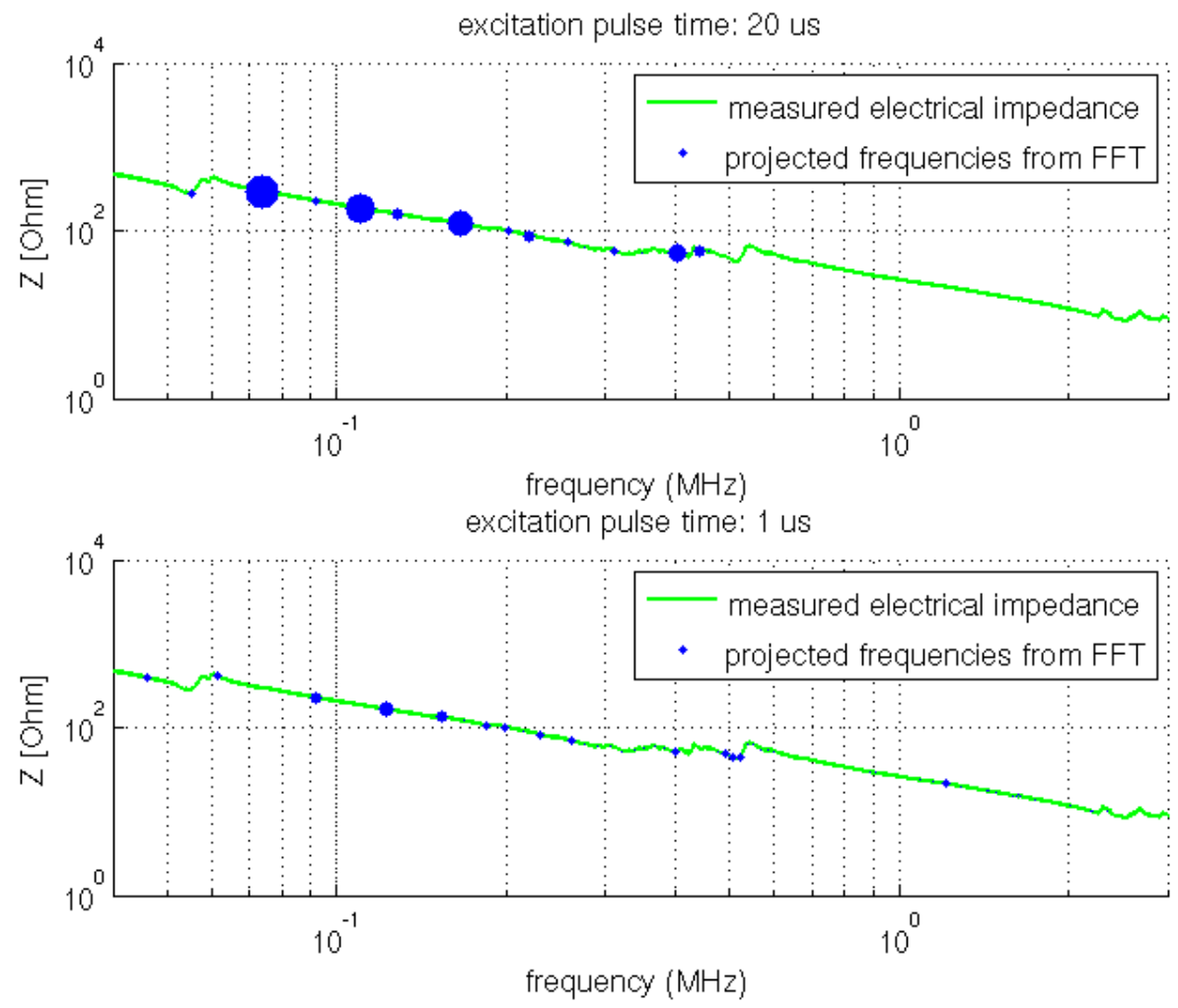

Figure 10. Comparison of the pressure wave frequencies after an excitation of $t_{\text {pulse }}=20 \mu \mathrm{s}$ (top) with those after $t_{\text {pulse }}=1$ $\mu \mathrm{s}$; the dots are the frequencies from the FFT, and their size corresponds to their number how often they occur.

\section{Acknowledgements}

We would like to thank the German Research Foundation (DFG) for financial support of this project.

\section{References}

[1] C. D. Rosebrock, N. Riefler, T. Wriedt, L. Mädler, and S. D. Tse. Disruptive burning of precursor/solvent droplets in flame-spray synthesis of nanoparticles. AIChE J., 59:4553-4566, 2013.

[2] O. Kurt, U. Fritsching, and G. Schulte. Secondary droplet formation during binary suspension droplet collisions. Atom. Spray, 19:457-472, 2009.

[3] O. A. Basaran, H. Gao, and P. P. Bhat. Nonstandard inkjets. Annu. Rev. Fluid Mech., 45:85-113, 2013.

[4] N. Riefler and T. Wriedt. Generation of monodisperse micron-size droplets using free adjustable signals. Part. Part. Syst. Charact., 25:176-182, 2008.

[5] S. Temple. Small fast inkdrop emission from a nozzle. Technical report, 43rd European Study Group with Industry, Lancaster University, 2-5th April 2002, 2002.

[6] H. Ulmke, T. Wriedt, and K. Bauckhage. Piezoelectric droplet generator for the calibration of particle-sizing instruments. Chem. Eng. Technol., 24:265-268, 2001.

[7] I. M. Hutchings and G. D. Martin, editors. Inkjet Technology for Digital Fabrication. Wiley, Chichester, 2012.

[8] F. Fahy and P. Gardonio. Sound and Structural Vibration. Elsevier, Academic Press, Amsterdam, 2006.

[9] F. A. Firestone. A new analogy between mechanical and electrical systems. J. Acoust. Soc. Am., 4:249-267, 1933. 